\title{
Ist Religion lehrbar? Diskurse über religiöse Bildung im spätantiken Christentum
}

\author{
Peter Gemeinhardt
}

Eingegangen: 4. März 2021 / Überarbeitet: 4. August 2021 / Angenommen: 25. August 2021 / Online publiziert: 24. November 2021

(C) Der/die Autor(en) 2021

Zusammenfassung Religion und Bildung stehen in einem Spannungsverhältnis zueinander, das fruchtbar, aber auch konflikthaft ausgestaltet sein kann. Das galt bereits für das frühe Christentum. Dem Befehl Jesu folgend galt es, Menschen vor und nach der Taufe Bildung zuteil werden zu lassen. Aber kann man Glauben „machen“? Und wenn ja, wie? Der Beitrag beleuchtet zunächst die Kritik paganer Philosophen am ,bildungslosen“ Christentum und dessen Ansätze zu religiöser Unterweisung. Er geht sodann auf das Bildungshandeln im Katechumenat des 4. und 5. Jahrhunderts ein und untersucht, wie das Lehren des Glaubens in didaktischer Hinsicht reflektiert wurde. Schließlich nimmt er eine anscheinend bildungsabstinente Gruppe in den Blick: das Mönchtum, das sich bei näherem Hinsehen als Bildungsbewegung sui generis entpuppt. Der Beitrag legt die Triangulation christlicher Bildung zwischen Lehrenden und Lernenden sowie Gott als eigentlichem Lehrer offen; er schließt mit Überlegungen, wie die spätantiken Impulse für modernes (religiöses) Bildungshandeln erschlossen werden können.

Schlüsselwörter Bildung · Religion · Katechumenat · Augustin · Spätantike

Im Quellenverzeichnis werden lediglich die verwendeten Übersetzungen aufgeführt. Die kritischen Editionen der herangezogenen Texte sind verzeichnet in Döpp und Geerlings (2002).

Prof. Dr. Peter Gemeinhardt $(\bowtie)$

Theologische Fakultät, Lehrstuhl für Kirchengeschichte, Georg-August-Universität Göttingen, Platz der Göttinger Sieben 2, 37073 Göttingen, Deutschland

E-Mail: peter.gemeinhardt@theologie.uni-goettingen.de 


\title{
On teaching religion: debates about religious education in late antique Christianity
}

\begin{abstract}
Religion and education coexist in a field of tensions; their interaction can be fruitful but also conflictuous. This was already true for early Christianity. According to Jesus' commandment, people should receive education before and after baptism. But can one give rise to faith? And if so, how? The present paper starts with pagan philosophers' criticism that early Christianity was, in their view, hostile towards education and with Christian attempts to establish a form of genuine religious instruction. In a second step, it examines educational procedures within the framework of the 4 th and 5 th century catechumenate and investigates how the teaching of the faith was subject to didactic reflection. After this, the paper focusses a Christian group that was apparently abstinent from education, namely, monasticism. However, a closer look reveals that the early monks formed an educational movement sui generis. Finally, the paper highlights the triangulation of Christian education between teachers, disciples, and God as the proper teacher; it closes with some thoughts on the possibility of adapting late antique ideas to modern discourses on (religious) education.
\end{abstract}

Keywords Education - Religion - Catechumenate $\cdot$ Augustine $\cdot$ Late Antiquity

\section{Einleitung: Kann man Religion lehren?}

Kann man Religion lehren? Wer, wie der Verfasser, an einer Theologischen Fakultät lehrt, ja sogar in BA- und MA-Studiengängen, die „Evangelische Religion“ heißen, wird dies kaum bestreiten. Ganz so harmlos ist die Frage aber nicht. Der Gegenstand der Theologie ist ja nicht zuerst Wissen über Religion, sondern die Reflexion religiöser Praxis, d.h. das Nachdenken über rituelle und intellektuelle Bezugnahmen auf eine außeralltägliche Wirklichkeit, die menschliches Leben umfassend bestimmt; so jedenfalls die Grundannahme, christlich gesprochen: der Glaube an den in der Geschichte wirkenden dreieinigen Gott. Dieser Gott agiert dabei so, dass er das Individuum nicht bedrückt, sondern dazu befreit, sich im Gegenüber zu jener Wirklichkeit, die jenseits aller Empirie vorgestellt wird, selbst zu bestimmen. Damit ist jeder Mensch zur Bildung seiner selbst berufen. Und so ist Religion nicht nur Gegenstand, sondern Grund von Bildung (vgl. Dressler 2006, S. 126). Das wiederum schließt nicht nur Glauben, sondern auch praktische Frömmigkeit ein, weil Religion ein ganzheitliches Geschehen ist, das - gerade weil es den ganzen Menschen ergreift - Anlass zur Selbst-Bildung gibt.

Daher ist die Eingangsfrage doppelt zu beantworten: Ja, Religion kann gelehrt werden, indem man kritisch eine Beobachterperspektive auf religiöse Lehr-LernProzesse einnimmt - und nein, Religion kann nicht gelehrt werden, weil sie als Bildungsprozess der externen Steuerung entzogen ist. So ist Religion für Bildung offen, sie kann (und muss) ihre Binnenlogik darlegen und kritisch reflektieren (lassen), nicht nur von externen Beobachtenden, sondern auch aus der Binnensicht. Inwieweit sich Religion aber auch Bildung verdankt, wie sich also Gottes Werk 
und des Menschen Beitrag bei der Begründung und Ausformung religiöser Bildung verhalten, darauf zielt die Frage: „Ist Religion lehrbar?“"1

Das Spannungsfeld von Bildung und Religion ist aktuell, aber nicht neu. ${ }^{2}$ Die Titelfrage meines Beitrags wurde seit Beginn des Christentums gestellt, und sie war offensichtlich dringlich. Dies soll hier für die formative Phase des Christentums - das erste halbe Jahrtausend seines Bestehens - beleuchtet werden. Dabei werde ich zunächst anhand der religiösen Unterweisung im frühen Christentum untersuchen, wie Katecheten das skizzierte Problem der Möglichkeiten und Grenzen des Lehrens von Religion lösten oder ob sie es auf sich beruhen ließen (I). Im zweiten Schritt diskutiere ich Ansätze einer christlichen Religionsdidaktik, die wir insbesondere bei dem einflussreichsten Theologen des lateinischen Abendlandes, Augustin von Hippo, finden (II), bevor ich nach alternativen Konzepten religiöser Bildung im monastischen Bereich frage (III). Abschließend formuliere ich eine These, inwieweit die Beantwortung aktueller Fragen nach dem Verhältnis von Bildung und Religion von der Analyse der Geschichte dieses Verhältnisses profitieren kann. ${ }^{3}$

\section{Religiöse Unterweisung im frühen Christentum: erst lehren, dann taufen!}

Wurde im entstehenden Christentum Religion für lehrbar gehalten? Offensichtlich ja! Religion wurde gelehrt: Juden und „Heiden“ wurden in der christlichen Religion unterwiesen, nach Erreichen eines gewissen Kenntnisstandes getauft und in die Gemeinschaft der Christen aufgenommen. Genau diesen Auftrag hatte der auferstandene Jesus, dem Evangelisten Matthäus zufolge, seinen Jüngern erteilt:

Gehet hin und lehret alle Völker: Taufet sie auf den Namen des Vaters und des Sohnes und des Heiligen Geistes und lehret sie halten alles, was ich euch befohlen habe (Mt 28, 19f.).

„Taufen“ ist hier gerahmt von ,in die Schule Nehmen“ (matheteuein) und „Belehren“(didaskein). Die Jünger, besser: „Schüler“ (mathetai) partizipieren an der Vollmacht ihres Herrn, indem sie dessen Auftrag in einem mehrstufigen Lehr-LernProzess umsetzen: Es bedarf einer vorgängigen Unterweisung, um die Taufe zu empfangen, doch folgt dieser weiteres Bildungshandeln. Die erste Unterweisung zielt auf eine willentliche Entscheidung des Menschen für den Glauben. Doch wird nach der

\footnotetext{
${ }^{1}$ Da der Begriff „Glaube“ im Deutschen eine Nähe zu kognitiven Vollzügen hat, verwende ich „Religion“ als umfassenderen Begriff, um zu verdeutlichen, dass im spätantiken Christentum (und auch seither) Glaubenserkenntnis stets mit Frömmigkeitspraxis in unterschiedlichen Institutionalisierungsgraden verbunden war.

2 Für eine Längsschnittdarstellung vgl. jetzt Gemeinhardt (2021b).

3 Im Hintergrund meiner Ausführungen stehen terminologische, kategoriale und inhaltliche Erträge der Arbeit des Göttinger Sonderforschungsbereichs „Bildung und Religion“ (2015-2020); dazu vgl. Gemeinhardt (2019 und 2020a) sowie zum Spannungsfeld von paganer und christlicher Bildung Gemeinhardt (2007). Eine Auseinandersetzung mit der reichlich vorliegenden Literatur zum Thema ist hier ausdrücklich nicht intendiert; als ein Beispiel, das über die Spätantike hinausführt, sei nur der Band Sautermeister und Zwick (2019) erwähnt.
} 
Initiation für eine vertiefte Form der Belehrung gesorgt. Im 4. Jahrhundert etablierte sich dafür der Begriff „Mystagogie“, deren Vorbedingung die Erfahrung des Ritus war. Die Einführung in die christliche Religion hatte also mit Bildung zu tun - das verband das Christentum mit dem Judentum, unterschied es aber von den öffentlichen und in diesem Sinne ,,politischen“ oder zivilreligiösen Kulten der griechischen und römischen Religion, die keine religiöse Belehrung vor der Initiation kannten. Allerdings entwickelte sich im selben Zeitraum - der Kaiserzeit (1.-3. Jahrhundert n. Chr.) - auch unter Philosophen, zumal im Mittel- und Neuplatonismus, eine Verbindung religiöser Praxis mit intellektueller Reflexion (Becker 2019), was (mit) erklärt, warum zwischen christlichen Theologen und nichtchristlichen Philosophen eine teils heftig ausgetragene Konkurrenz herrschte (s. unten).

Eine Taufvorbereitung gab es offenbar schon früh, erst nach 200 n. Chr. ist aber ein institutionalisierter Katechumenat zu beobachten (vgl. Metzger et al. 2004). Eine Kirchenordnung, die Traditio apostolica, bietet für Rom Einblicke in Ablauf, Akteure und Ziele (Gavrilyuk 2007, S. 96 ff.) ${ }^{4}$ : Am Anfang stand eine Prüfung der Motivation und des Lebenswandels des Taufwilligen. So konnte nicht Christ werden, wer ein Bordell betrieb, im Theater auftrat oder Gladiator war, aber auch wer in der Grammatik- oder Rhetorikschule unterrichtete, allerdings war hier eine Ausnahmegenehmigung möglich (Traditio apostolica 16). Die Unterweisung dauerte bis zu drei Jahre, an deren Ende geprüft wurde, ob die Aspiranten ,während des Katechumenats ehrbar gelebt, die Witwen unterstützt, Kranke besucht, ob sie also alle Arten von guten Werken getan haben“ (Traditio apostolica 20). Der frühchristliche Katechumenat umfasste demnach ethische und lebenspraktische Aspekte, schloss aber auch die Kompetenz ein, die Tauffragen zu beantworten, die später als Glaubensbekenntnis gefasst wurden. Man musste kein Theologe sein, um Christ werden zu können; aber Neugetaufte sollten nicht nur wissen, wie sie zu leben, sondern auch, was sie zu glauben hatten, und diesen Glauben aus freien Stücken bekennen. Darum erging am Schluss der Taufzeremonie an jeden einzelnen frisch gebackenen Christenmenschen die Mahnung,

gute Werke zu tun, Gott zu gefallen, sich eines guten Lebenswandels zu befleiBigen, voll Eifer sich an die Kirche zu halten, das zu tun, was er gelernt hat, und in der Frömmigkeit voranzuschreiten (Traditio apostolica 21).

Die Taufe war demnach der Schritt, der gegangen werden sollte, nachdem die christliche Religion umfassend und tiefgreifend vermittelt worden war. Die Aufnahme in die Gemeinde ohne vorausgehende Belehrung war theologisch nicht denkbar. Religion galt als lehrbar - und wurde gelehrt.

In der frühchristlichen Mission ging Bildung auch deshalb notwendigerweise der Taufe voraus, weil eine Sozialisation in christlichen Familien nur sehr allmählich die Regel wurde (auch wenn faktisch schon in dieser Zeit Kindertaufen vorkamen). In dieser Minderheitensituation wurde neben die Unterweisung der Taufwilligen die Auseinandersetzung mit Kritikern dieser neuen religiösen Formation wichtig. Schematisch gesprochen, trat neben die Katechetik die Apologetik. Damit stand man aber in Konkurrenz zu anderen Bildungstraditionen: Paulus griff auf dem Areopag in

\footnotetext{
4 Übersetzungen nach Geerlings (1991).
} 
Athen (Apg 17, 16-34) die hellenistische Philosophie auf, um die Athener davon zu überzeugen, dass sie unwissentlich längst den christlichen Gott verehrten. Der Erfolg war überschaubar, aber die Herausforderung, sich in einem hoch pluralisierten religiösen Kosmos mit einer exklusiven Botschaft zu positionieren, war angenommen. Die Apologeten des 2. Jahrhunderts gingen einen Schritt weiter und präsentierten das Christentum als passfähig zum philosophischen Denken, ja als ,einzige verlässliche und nutzbringende Philosophie“ (Justin, Dialogus cum Tryphone 8,1). Justin $(\dagger 165)$, der Leiter einer christlichen Philosophenschule in Rom, argumentierte, recht verstandene Bildung müsse zur Einsicht in die Wahrheit der christlichen Lehre führen - oder wenigstens die Obrigkeit veranlassen, die Verfolgungen von Christen einzustellen, verstand sich ein Kaiser wie Marc Aurel (161-180) doch als „Philosoph“ und „Bewunderer der Bildung“ (Justin, 1 Apologia 1). ${ }^{5}$

Dieses Selbstbewusstsein konnte als Provokation aufgefasst werden. So warf der Platoniker Celsus in einer um 180 verfassten Streitschrift den Christen einen ,unvernünftigen“ Glauben vor (zit. bei Origenes, Contra Celsum 1,9). ${ }^{6}$ Die Christen als selbsternannte Philosophen verzichteten in dieser Sicht auf die Vermittlung, ,philosophischer" (vernunftgemäßer) Bildung und verlangten stattdessen uneingeschränkte Zustimmung zu ihrer Lehre (Contra Celsum 3,39), predigten also einen Fideismus. In der Tat betonte nur wenig später Clemens von Alexandrien ( $\dagger$ ca. 215 ), Glaube beinhalte die freiwillige (!) Zustimmung zu Lehren (dogmata) und sei daher ,ein Akt des Wählens“ (prohairesis), eine Entscheidung für oder gegen das Wort Gottes, das einzigartige Autorität beanspruche (Clemens von Alexandrien, Stromata 2,9,2f.; $2,11,1 ; 2,27,4)$. Auch Clemens' jüngerer Zeitgenosse Origenes $(\dagger 253)$ wies den Vorwurf eines dezisionistischen Glaubenszwanges zurück:

Wenn es möglich wäre, dass sich alle von den Aufgaben des Lebens freimachten und Zeit zum Philosophieren hätten, dürfte niemand einen anderen Weg einschlagen als diesen allein ... Hinsichtlich der Menge der Gläubigen, die sich der Fülle des Lasters, in der sie sich früher wälzten, entledigt haben, fragen wir, was besser für sie ist: unreflektiert zu glauben, aber ihr sittliches Leben in irgendeiner Weise geordnet zu haben ... oder ihre mit schlichtem Glauben verbundene Bekehrung solange nicht zuzulassen, bis sie sich einer Prüfung der Lehren widmen können? (Origenes, Contra Celsum 1,9).

Origenes räumte ein, es sei ,auch nach Überzeugung der Schrift besser, mit Vernunft und Weisheit den Lehrsätzen zuzustimmen als nur mit einfachem Glauben“ (Contra Celsum 1,13). Doch konnte der philosophische Diskurs nicht als Maßstab für das Lehren von Religion allgemein dienen, vielmehr verteidigte Origenes die christliche Praxis, allen Menschen die Teilhabe an dieser Religion zu eröffnen, auch denen, die nicht als intellektuell satisfaktionsfähig galten oder keine Stimme in der Öffentlichkeit hatten (vgl. Contra Celsum 3,44-58). Origenes konzentrierte sich in seinen Schulen in Alexandrien und später in Caesarea in Palästina zwar auf die Vermittlung umfassender Kenntnisse in Philosophie und Theologie an wenige dazu Befähigte; seine Lehrinstitution ähnelte einer „Privatuniversität“ (Markschies 2007,

\footnotetext{
5 Übersetzung nach Ulrich (2021).

6 Übersetzungen aus Contra Celsum: Barthold (2011).
} 
S. 101). Als Bildungsvermittler war er aber kein Solitär: Seine Antwort auf Celsus' Kritik am „,blinden Glauben“ zeigt, dass auch grundsätzlich im Christentum nicht auf Bildung verzichtet wurde, sondern dass diese im Gegenteil allen Interessierten angeboten, ja zugemutet wurde. Pointiert ausgedrückt: Das Christentum war eine Bildungsreligion, aber nicht nur die Religion der Gebildeten!

Offensichtlich konkurrierten im 2. und 3. Jahrhundert nicht nur verschiedene Formen von Religion und ihrer Reflexion, sondern auch unterschiedliche Konzepte von Bildung. Für das frühe Christentum war daher von Anfang an die Frage akut, wie man es mit den Idealen der Enkyklios Paideia bzw. der Bonae Artes hielt, d. h. mit dem Inbegriff von Bildung der römischen Oberschicht (Vössing 2003). Die Schultexte - besonders die Epen Homers - wurden als ,heidnisch“ bezeichnet, da sie von zahlreichen Göttern erzählten, die aber (aus christlicher Sicht) falsche Götter waren! Theologen wie die oben genannten Alexandriner Clemens und Origenes meinten, man müsse die antike Paideia als ,Vor-Bildung“ für das Christentum fruchtbar machen. Einigkeit herrschte unter allerdings - soweit uns die Texte christlicher Autoren erhalten sind -, dass formale schulische Unterweisung in Grammatik und Rhetorik oder gar philosophische Gelehrsamkeit keine Vorbedingung war, um zu einer Gemeinde gehören zu können. Im Gegenteil: Der Apologet Tertullian († nach 220) warnte, dass man in den scholae publicae zwangsläufig mit „heidnischer“ Religion in Berührung komme; zumal die Lehrer der höheren Bildungsstufen (professores litterarum) könnten dies schlechterdings nicht vermeiden:

Es gibt keinen Zweifel, dass sie mit vielerart Götzendienst in Verbindung stehen; denn sie müssen die heidnischen Götter verkünden, ihre Namen und die Abstammung nennen, die jeweiligen Mythen und Ehrenattribute erklären, schließlich müssen sie deren Feste und Feiertage beachten, an denen sie ja ihren Lohn erhalten (Tertullian, De idololatria 10,1). ${ }^{7}$

Für Tertullian war ein Lehrer seinem Gegenstand zu Loyalität verpflichtet; der exklusive Anspruch der christlichen Religion wurde dadurch kompromittiert. Er konzedierte, Lernende könnten eine hinreichende Distanz zum Gegenstand einnehmen, wenn sie frühzeitig dafür sensibilisiert würden. Lehrenden sei es aber nicht möglich, Klassiker wie Vergil als reine Literatur zu behandeln, und daher sei eine schwerwiegende Kontamination praktisch unvermeidlich:

Wenn der Gläubige Lesen und Schreiben lehrt, dann empfiehlt er dabei ohne Zweifel die (in den Texten) eingestreuten Preisungen der Götter; dadurch, dass er sie weitergibt, bekräftigt er sie, und indem er sie erwähnt, legt er Zeugnis für sie ab. Die Götter bezeichnet er mit eben diesem Namen, obwohl doch das Gesetz verbietet, sie Götter zu nennen und diesen Namen grundlos zu gebrauchen. So wird der Grund zum Glauben an den Teufel schon mit den Anfängen der Bildung gelegt. Nun untersuche noch lange, ob Götzendienst (idololatria) treibt, wer über die Götzen unterrichtet (de idolis catechizat)! (De idololatria 10,5f.).

Tertullian betrachtete das Schulgeschehen also - in anachronistischer Begrifflichkeit - als konfessionsgebundenen Religionsunterricht: Der Begriff catechizare

7 Übersetzungen nach Vössing (1997, S. 304 ff.). 
erinnert kaum zufällig an den innerchristlichen Katechumenat! Er betonte aber völlig zurecht, dass sich ein Lehrer, wenn er seine Aufgabe erfüllen und dafür bezahlt werden wolle, nicht kontinuierlich von seinem Lehrgegenstand distanzieren könne. Dies wurde allerdings in seinem Umfeld nicht in derselben Schärfe gesehen; die einzige vergleichbare spätantike Äußerung stammt von dem dezidiert ,heidnischen“ Kaiser Julian (361-363), der Christen am Lehren hindern wollte, eben weil sie den Göttern die schuldige Verehrung verweigerten (Epistula 61c; vgl. Gemeinhardt 2021a, S. 161-164). Tertullian und Julian äußerten damit von diametral verschiedenen Ausgangspunkten aus praktisch dieselbe Außenseitermeinung (vgl. Gemeinhardt 2008). Der Gang der Geschichte sollte freilich, was das Verhältnis von Bildung und Religion anbetraf, ein anderer sein.

\section{Planvoll zu Werke gehen: Didaktische Reflexion religiöser Unterweisung}

Mit der „Konstantinischen Wende“ zu Beginn des 4. Jahrhunderts änderte sich vieles für die christliche Kirche - schon damals sagten manche Zeitgenossen: zum Schlechteren. Zwar war die von Kaiser Konstantin († 337) begonnene Förderung des Christentums dadurch vorbereitet, dass dieses sich schon im 3. Jahrhundert als gesellschaftliche Kraft etabliert hatte; es gab, wie das Beispiel Julians (s. oben) zeigte, auch keine Garantie, dass die Verfolgungen ein für allemal Geschichte sein würden. Aber die seit Konstantin sukzessive wachsende Bedeutung des Christentums auf dem Markt spätantiker Religiosität wirkte sich auf viele Lebensbereiche aus, auch auf die Art und Weise, wie man auf das Christ-Werden vorbereitet wurde. Ein mehrjähriger Katechumenat - wenn er vorher überhaupt die Regel gewesen war - gehörte bald der Vergangenheit an. „Katechumenen“ waren nun diejenigen, die sich ohne formalisierte Zugehörigkeit im Dunstkreis einer Gemeinde befanden und die Gottesdienste besuchten, sich aber noch nicht zur Taufe entschlossen hatten. Dieser Zustand konnte lange anhalten, mitunter wurde die Taufe erst auf dem Sterbebett erteilt, wofür Kaiser Konstantin höchstselbst ein Beispiel war (Euseb von Caesarea, De vita Constantini 4,62,4f.). Erst wer sich zur Taufe anmeldete, erhielt in der vorösterlichen Fastenzeit einen Crashkurs in christlicher Glaubenslehre. So wurden aus „Katechumenen“ (,Unterwiesenen“) „Photizomenoi“ (,Erleuchtete“, so Kyrill von Jerusalem, Procatechesis 1). Was blieb, war die Forderung nach einer unumkehrbaren Entscheidung: Nach der Anmeldung zur Taufe gab es keinen Weg zurück.

Der Weg zu dieser Entscheidung konnte ganz unterschiedlich sein. Der römische Rhetor Marius Victorinus ( $\dagger$ um 365) studierte lange und intensiv die Bibel und erklärte schließlich seinem Freund Simplicianus: „Du magst es wissen: Ich bin schon Christ" (Augustin, Confessiones 8,2,4). ${ }^{8}$ Dieser konnte das so nicht stehen lassen: „Ich möchte Dich nicht als Christ unter Christen zählen, bevor ich Dich in der Kirche Christi gesehen habe.“ Worauf ihm Victorinus entgegnete: ,Sind es also die Kirchenwände, die den Christen machen?" Der Dialog, den Augustin in den Confessiones

\footnotetext{
8 Übersetzung nach Bernhart (1987).
} 
überliefert, endete hier - aber nur vorläufig: Schließlich entschloss sich Victorinus doch noch zur Taufe, ,zum Staunen Roms, zur Freude der Kirche“. Sein Weg zum Christentum war der eines Autodidakten, der nach seiner intellektuellen Auseinandersetzung mit dem christlichen Glauben auf ein öffentliches Bekenntnis verzichten zu können glaubte. Das allerdings war seinerzeit eine Ausnahme, und Augustin berichtete davon, um zu verdeutlichen, dass auch ein individueller Bildungsprozess zur regulären Taufe führen musste. Dass mit Victorinus ein gefeierter Vertreter der Elitenbildung zum Christentum konvertierte, unterstrich dessen Annehmbarkeit, ja Attraktivität für die römische Oberschicht.

Victorinus' Bekehrung vollzog sich in den 350er Jahren, und in dieser Zeit wird die Quellenlage zum Katechumenat besser. Erhalten sind mehr oder weniger umfangreiche Reihen von Taufkatechesen von Kyrill von Jerusalem $(\dagger 387)$, Johannes Chrysostomus $(\dagger 407)$ und Theodor von Mopsuestia $(\dagger 428)$ sowie von Ambrosius von Mailand (†397) und Nicetas von Remesiana ( $†$ nach 414), weiterhin katechetische Predigtreihen von Zeno von Verona ( $†$ nach 370) und Augustin ( $†$ 430). Diese Textcorpora sind in der Forschung intensiv untersucht worden (Gavrilyuk 2007, S. 163 ff.; Harmless 2014, S. 35 ff.; Paul 1993, S. 75 ff.), doch bleibt zu beachten, dass der Katechumenat an vielen Orten nur in kleinen Ausschnitten dokumentiert ist - obwohl sich in dieser Zeit die „Heiden“, so Bischof Gaudentius von Brescia († um 410), „mit der Schnelligkeit eines rollenden Rades“ bekehrten (Sermo $8,25)$. Die grundlegende Belehrung über den christlichen Glauben und die aus ihm entspringende Frömmigkeitspraxis beschränkte sich offenbar auf die unmittelbare Taufvorbereitung. Jedoch wurde christliches Glauben und Handeln in jedem Gottesdienst thematisiert; Christen blieben daher lebenslang Lernende, auch wenn das Niveau dieser Unterweisung im Übergang zum Frühmittelalter vielerorts eher gering zu veranschlagen sein dürfte (Kinzig 2019).

Die Grundstruktur des Missionsbefehls Jesu blieb damit maßgeblich: Die Taufe wurde von Belehrung eingerahmt. Je spärlicher die Unterweisung vor der Taufe ausfiel, umso dringlicher stellte sich das Problem der katechetischen Nacharbeit, denn seit dem 5. Jahrhundert fanden im Zuge der sogenannten Völkerwanderung in Gallien und anderen Regionen mitunter Massentaufen statt, denen sozusagen die Christianisierung der Christen oft erst folgte. Mit dem Ausgreifen des Christentums über die Städte hinaus in ländliche Gebiete nahm die Bedeutung der Presbyter gegenüber den Bischöfen zu, weshalb die Unterweisung der Gläubigen zunächst eine Ausbildung der Kleriker erforderte; diese blieb in Spätantike und frühem Mittelalter allerdings rudimentär (Patzold 2015).

Jedoch sollte aus den genannten Entwicklungen nicht der Schluss gezogen werden, seit der „Konstantinischen Wende“ seien der Glaubensernst der Christen im Allgemeinen und ihre Bildungswilligkeit im Besonderen im Sinkflug begriffen gewesen. ${ }^{9}$ Bemerkenswert ist vielmehr, dass auch unter gewandelten Bedingungen der Anspruch auf individuelle Glaubenseinsicht erhalten blieb: Katechetische Bildung zielte auf Glaubenswissen sowie auf ethisch verantwortliches Verhalten und mit beidem auf Partizipation an der communio sanctorum. Es ging ,in erster Linie

\footnotetext{
9 So das in der angloamerikanischen Welt einflussreiche Buch von Dujarier (1979, S. 79): „With the ease of the Constantinian era, quality unfortunately gave way to quantity."
} 
[um die] Initiation in einen Lebensstil“" (Schröder 2012, S. 50) - und zwar in einen „way of life“, der selbstverantwortet gestaltet werden sollte. Hierin kann der rote Faden christlicher Bildungsbemühungen in der Spätantike erblickt werden.

Dies zeigen auch die (insgesamt wenigen) Texte, die solche Bildung didaktisch reflektierten. Hierfür spielte oft die Metapher des Bauwerks eine Rolle. ${ }^{10}$ So erklärte Kyrill von Jerusalem $(† 387)$ in seinen im Jahr 351 gehaltenen Katechesen den frisch eingeschriebenen Taufbewerbern:

Denk dir, der katechetische Unterricht ist ein Gebäude! Wenn wir nicht tief gegraben und einen Grund gelegt haben, wenn wir nicht planmäßig ein wohlgefügtes Gebäude errichtet haben, damit sich keine Risse zeigen und der Bau nicht Schaden leide, dann ist auch die anfängliche Mühe wertlos ... Viele Lehren sind es, welche planmäßig vorgetragen werden ... Wenn du nicht die Lehren zu einem Ganzen zusammenfügst und die früheren zugleich mit den späteren im Gedächtnis behältst, dann wird dein Bau Risse zeigen trotz der Arbeit des Baumeisters (Kyrill von Jerusalem, Procatechesis 11). ${ }^{11}$

Im Bild fungierte also der Katechet als Baumeister, während die Katechumenen sozusagen die Bauhandwerker ihrer eigenen religiösen Behausung waren. Aber nicht nur menschliche Lehrende und Lernende waren involviert, sondern auch Christus selbst: Gebe es doch, so Kyrill, neben dem ,dogmatischen“ Glauben, dem Gebäude aus lehrhaften Sätzen, das kognitiv vermittelt und angeeignet werden konnte, den Glauben als „Gnadengeschenk“, der Berge versetzen könne und die Seele zur Gottesschau befähige (Kyrill von Jerusalem, Catecheses baptismales 5,10f.). Das intellektuelle Lernen des Glaubens wurde durch eine geistliche Gabe ergänzt. Für Kyrill war Religion demnach zum Teil von Menschen lehrbar - und zum Teil gottgegeben.

Didaktisch reflektiert wurde diese Doppelperspektive bei Augustin, auf den hier etwas ausführlicher eingegangen werden soll. In den „Bekenntnissen“ (Confessiones), die er gut zehn Jahre nach seiner 386 erfolgten Bekehrung schrieb, präsentierte sein eigenes Leben als umwegreiche Bildungsbiographie: Er musste sich hiernach die Wertschätzung biblischer Bildung erst gegen die fatale „Ver-Bildung“ durch den Schulunterricht erkämpfen. Doch blieben die dort erworbenen Kenntnisse in seinem Werk immer präsent (immerhin war er anderthalb Jahrzehnte lang Grammatik- und Rhetoriklehrer gewesen). Das zeigt schon sein erstes Bildungsprogramm: Gleich nach seiner Hinwendung zum Christentum entwarf Augustin eine christliche Neubegründung des Curriculums der artes liberales. Deren Kenntnis würde, Schritt für Schritt hinansteigend, zur Erkenntnis der Wahrheit führen. Der Ort dieses stufenförmigen Weges, auf dem Religion gelernt werden kann, ist nicht eine asymmetrische Unterrichtssituation, sondern der Dialog der Gebildeten. Den Anfang macht allerdings die auctoritas, hier: die Autorität der Bibel, der man Vertrauen oder - das lateinische Wort fides gibt beides her - Glauben schenken muss (De ordine 2,9,26f.). Wie bei Kyrill ist nicht nüchternes Fürwahrhalten, sondern Existenz bestimmendes

\footnotetext{
10 Vgl. Johannes Chrysostomus (De inani gloria et de educandis liberis 23-25, sowie Kap. 22 zum Vergleich der Kinder mit Statuen, die von den Eltern wie von Bildhauern modelliert werden).

11 Übersetzungen nach Haeuser (1922).
} 
Vertrauen die Grundlage, auf der die ratio selbsttätig durchdringt, was die auctoritas lehrt (vgl. Gemeinhardt 2021a, S. 169). So steigt man über Wissenschaft und Wahrheit zur Weisheit empor - und lernt am Ende, so Augustin, dass Gott ,,besser durch Nichtwissen gewusst wird" (scitur melius nesciendo; De ordine 2,16,44). Aber nur wer von Wissen zu Wahrheit und Weisheit gelangt ist, weiß, worüber nicht mehr $\mathrm{zu}$ sprechen und wovon daher zu schweigen ist. Dieses reflektierte Nichtwissen ist Ergebnis eines Prozesses, der den Menschen zu Gott und damit zu sich selbst als Geschöpf Gottes führt - es ist also Bildung.

Dieses Bildungsideal hatte seinen Ort im elitären Kreis christlicher Philosophen auf dem Landgut Cassiciacum in der Nähe von Mailand - ein Ort der Muße (otium) abseits des Alltagsgeschäfts (negotium). Die umfassende ,weltliche“ Bildung, die alle Mitglieder dieser Lebens- und Lerngemeinschaft erworben hatten (mit Ausnahme von Augustins Mutter!), wurde dabei in ein Ideal gelehrter geistlicher Existenz integriert, das in manchen Zügen an Origenes' Konzept für seine Lehrinstitution erinnerte (s. oben). Doch nachdem Augustin in seine Heimat Nordafrika zurückgekehrt und Bischof geworden war, musste er anders vorgehen. Die Vielfalt an Lebensund Glaubensweisen in seiner volkskirchlichen Gemeinde war die zentrale Herausforderung, der er mit katechetischem Handeln zu begegnen suchte. Dabei konnte bei den meisten Christinnen und Christen oder solche, die es werden wollten, nicht an schulische Bildung angeknüpft werden. Das führte Augustin von der Synthese zur Antithese, die sich auch in den Confessiones niederschlug (s. oben) und sich wie folgt skizzieren lässt: Gelernt werden muss, aber ,in der Schule des himmli-

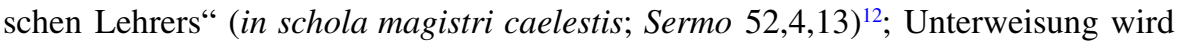
erteilt, aber nicht in der ,weltlichen“ Literatur, sondern in der „Literatur Christi“, der Bibel (Sermo 270,1). Deren Botschaft wird wiederum auf einen klaren Markenkern zugespitzt: Die Katechumenen sollen lernen, „Gott um Gottes willen und den Nächsten um Gottes willen zu lieben“ (De doctrina christiana 2,7,10). ${ }^{13}$ Es gilt, das innere Auge zu reinigen, das zur Gottesschau fähig ist, und zu einem „Heiligen“ zu werden, was für Augustin heißt: zu einem Menschen, der Weisheit (sapientia) durch Gottesnähe erlangt hat (De doctrina chr 2,7,11). Der intellektuelle Aufstieg zu Gott beginnt nicht mit Schulbildung, sondern mit dem Doppelgebot der Liebe (Mt 22,37-39); und daher ist die Bibel für Gläubige (und noch nicht Gläubige) so zu erschließen, dass sie zum Zeugnis des liebevoll zuvorkommenden Heilshandelns Gottes wird.

Was aber konnte ein Bischof und Lehrer „machen“, um Menschen zu solcher Bildung anzuleiten? Zwar ließ sich die Erleuchtung der Gläubigen nicht methodisch herbeiführen, aber der Katechet konnte und sollte den Boden für den Aufstieg zu Gott bereiten. Darum war das Lehren von Religion eine Kunst, die geübt werden wollte. Augustin ließ an der Grenzziehung gegen „heidnische“ Bildung keinen Zweifel aufkommen, zog allerdings nicht mit so scharfen Invektiven dagegen zu Felde wie sein Zeitgenosse Hieronymus $(† 419)$ : Die in der Schule erlernten Wissensbestände und Kompetenzen konnten unter entsprechenden Vorsichtsmaßnahmen

\footnotetext{
12 Vgl. Studer (1996) sowie Augustin (De civitate dei 22,30) zum Ideal des christlichen eruditus et doctus; zu Christus als Lehrer vgl. Gemeinhardt (2020b, S. 150-161).

13 Übersetzungen aus De doctrina christiana nach Pollmann (2002).
} 
rezipiert werden, so wie die Israeliten beim Exodus das Gold und Silber der Ägypter mit sich geführt hatten (Ex 3,21f.; 12,35f.). Augustin verstand dies allegorisch:

So bestehen alle Wissenschaften der Heiden nicht nur aus vorgetäuschten und abergläubischen Erdichtungen und schweren Bürden von überflüssiger Mühe, die jeder einzelne von uns unter der Führung Christi bei seinem Auszug aus der Gemeinschaft der Heiden verwünschen und meiden muß, sondern sie enthalten auch die sogenannten freien Künste, die für den Nutzen der Wahrheit recht geeignet sind, und einige sehr nützliche Vorschriften zur Lebensführung. Selbst über die Verehrung des einzigen Gottes findet sich bei ihnen einiges Wahre. Dies muß ein Christ, während er sich geistig von deren elenden Gesellschaft trennt, von ihnen zum rechten Gebrauch der Verkündigung des Evangeliums wegtragen, gleichsam wie ihr Gold und Silber, das die Heiden nicht selbst eingerichtet haben, sondern sozusagen aus den Minen der alles durchdringenden Vorsehung ans Tageslicht gebracht und auf verquere und unrechte Weise mißbraucht haben, um Dämonen zu willfahren (Augustin, De doctrina christiana $2,40,60)$.

Entscheidend war also die kritische Rezeption und konstruktive Ingebrauchnahme von Bildung zu religiösen Zwecken. Wie sollte das konkret vonstatten gehen? Augustin diskutierte in De doctrina christiana die Bedeutung der Rhetorik für die Predigt. Gewiss muss sich ein Lehrer verständlich machen können, und das ist das Ziel der Rhetorik (De doctrina christiana 4,13,29). Aber diese muss kritisch, d.h. nicht zum Selbstzweck, eingesetzt werden - dann ist sie allerdings unentbehrlich. Cicero († 43 v. Chr.) liefert die Maxime dazu: ,Weisheit ohne Beredsamkeit nützt wenig, Beredsamkeit ohne Weisheit schadet meistens und nützt niemals“ (Cicero, De inventione 1,1,1; zit. in Augustin, De doctrina christiana 4,5,7). Wer Religion lehren will, muss diese Weisheit selbst ergriffen haben, muss aus eigener Glaubenserfahrung, nicht abstrakt, reden. Aber Weisheit, die nicht didaktisch reflektiert wird, bleibt wiederum fruchtlos. Zugespitzt gesagt: Mag ja sein, dass der Geist weht, wo er will (Joh 3,8), sicher auch auf der Kanzel. Wo aber ein Katechet verlässlich Religion lehren soll, macht Übung den Meister!

Augustin hatte aber nicht nur den Lehrer, sondern auch die Lernenden im Blick; das macht den besonderen Reiz seines Bildungskonzepts aus. In De catechizandis rudibus lieferte er eine praktische Anleitung, wie man Anfänger belehrt. Ein Diakon hatte Augustin um Rat gebeten, wie er Interessierte ins Christentum einführen könne, und zwar so, dass die Bewerber das Richtige lernten und bei der Sache blieben und er selbst sich die Freude am Lehren bewahre. Wie feinfühlig Augustin analysiert, warum dem Katecheten bisweilen die rechte Disposition fehle, wie die Diskrepanz zwischen intellektueller Erkenntnis und ungenügendem sprachlichem Ausdruck zu ertragen sei und wie man dem Wegdämmern der Zuhörer durch praktische Maßnahmen begegne (z. B., indem man ihnen von Anfang an eine Sitzgelegenheit anbietet; De catechizandis rudibus 13,19$)^{14}$ - das macht diese Schrift zu einem Kabinettstückchen religionsdidaktischer Praxis (Reil 1989).

14 Übersetzungen nach Steinmann und Wermelinger (1985). 
Religion zu lehren erfordert ein gerüttelt Maß an säkularer Bildung auf Seiten des Katecheten; anders das Lernen von Religion, bei dem es darauf ankommt, wen man vor sich hat und auf welchen Voraussetzungen man aufbauen kann: Die Vertrautheit mit Grammatik und Rhetorik, also mit rein schulischer Bildung, führt einen Menschen mit ziemlicher Sicherheit zur Geringschätzung der Bibel, wie Augustin aus eigener Erfahrung weiß - solchen Leuten sei freundlich, aber bestimmt ihre „Ver-Bildetheit" aufzuzeigen (De catechizandis rudibus 9,13). Wenn sich dagegen ein philosophisch Kundiger über das Christentum informieren wolle, könne an sein philosophisches Wissen angeknüpft werden (so wie für Augustin selbst die Lektüre der Libri Platonicorum den Weg zur Hochschätzung der Bibel gebahnt hatte; Confessiones 7,9,13). Maßgeblich bleibt aber das Vertrauen auf die richtige Lehrautorität, nämlich auf die Bibel (De catechizandis rudibus 8,12). Denn, so Augustin in einer Predigt: „Die Seele möge es nicht wagen, etwas über Gott in ihrer eigenen Phantasie zu erdenken: Wer finden will, wird nicht finden, bevor er nicht gelernt hat" (Sermo Dolbeau 25,7).

Augustin macht in De catechizandis rudibus Religion zum Gegenstand einer fachgerechten didaktischen Analyse, indem er erläutert, ,welche Wissensinhalte wem, von wem, in welcher Art und Weise und unter welchen Umständen zu vermitteln sind“" (Reil 1989, S. 55). Dabei entsprechen sich die Sache, die zu vermitteln ist, und die Haltung, die der Vermittelnde einnehmen soll: Wie Gott die Menschen geliebt hat, so möge der Katechet seine Hörer lieben, damit diese ,vom Hören zum Glauben, vom Glauben zur Hoffnung, von der Hoffnung zur Liebe“ gelangen (De catechizandis rudibus 4,8). Wenn und insofern der Lehrer die Liebe selbst versprüht, von der er spricht, redet er nicht über Religion, sondern von dem, was er selbst erkannt hat, indem es ihn selbst ergriffen hat. Religion zu lehren ist für Augustin eine Sache persönlicher Beteiligung, sie kann nicht distanziert, quasi „konfessionsneutral“ gelehrt werden. Vielmehr ist sie nur an den Mann oder an die Frau zu bringen, wenn der Lehrende den Lernenden nicht überlegen, routiniert oder gar gelangweilt begegnet, sondern Liebe ausstrahlt - Liebe im Sinne des antiken Ideals der „Freundschaft"“ (amicitia), auf eine höhere Stufe gehoben durch die Erkenntnis, dass zuerst Gott dem Menschen als Freund begegnet. So schuldet nun der Mensch dem Menschen, der Katechet dem Katechumenen, ihn aus Liebe zu Gott mit Gott als dem Grund und Ziel christlicher Bildung bekannt zu machen (De catechizandis rudibus 12,17). „Freude“ (hilaritas) ist gefordert - und wird zugleich und zuerst von Gott selbst geschenkt:

„Einen freudigen Geber hat Gott lieb“ (2 Kor 9,7). - Dass sich aber diese freudige Stimmung im entscheidenden Augenblick einstellt, liegt bei der Barmherzigkeit dessen, der sie gefordert hat (De catechizandis rudibus 2,4).

Katechet und Katechumene stehen demnach gemeinsam vor Gott, und wenn der Lernende „durch unseren Mund Gott zuhört“ (De catechizandis rudibus 7,11), dann wirkt dies auch auf den zurück, der mit dem Lehren von Religion betraut ist:

Wenn die Zuhörer dieses Gefühl (sc. die freundschaftliche Liebe) uns gegenüber empfinden, die wir sprechen, wir aber ihnen gegenüber, die lernen, dann sind wir gleichsam gegenseitig Mitbewohner, und was jene hören, das sprechen 
sie gleichsam in uns, und wir lernen gewissermaßen in ihnen, was wir lehren

(De catechizandis rudibus 12,17).

Religion ist lehrbar, so lässt sich zuspitzen, weil Gott selbst lehrt - aber nicht ohne menschliches Zutun. Sprachliche Präzision ist daher auch unter Christen keineswegs gleichgültig: „Das Volk soll ja sein ,Amen` zu etwas sagen können, was es völlig versteht" (De catechizandis rudibus 9,13). Aber im gelingenden Katechumenat werden Lehrende und Lernende im Gegenüber zu Gott einander zu Dialogpartnern, unter denen der Lehrer allenfalls einen relativen Vorsprung an Bildung besitzt. Das gilt für die Einführungskatechese unter vier Augen wie für den Gottesdienst der Gemeinde: Diese ist, so Augustin, die schola Christi, in der sichtbar und hörbar der Bischof lehrt, durch den aber tatsächlich Christus als Autorität agiert:

Christus ist es, der lehrt; sein Katheder steht im Himmel ... Seine Schule befindet sich auf der Erde, und seine Schule ist sein Leib. Das Haupt lehrt seine Glieder (De disciplina Christiana 15).

\section{Ohne menschliches Zutun? Göttliche Erziehung im frühen Mönchtum}

Die religionsdidaktischen Überlegungen Augustins richten sich auf eine ,,volkskirchliche" Situation: auf eine Mischung aus überzeugten, manchmal in ihrer Überzeugung schwankenden Christinnen und Christen und aus Menschen, die erst noch Katechumenen werden mussten. Zugleich hatten aber auch alternative Formen christlichen Lebens Konjunktur, damit auch besondere Konfigurationen christlicher Bildung, und zwar an Orten, wo man es nicht erwarten würde: unter den Vätern (und Müttern) der ägyptischen Wüste (eremos), den „Eremiten“, und denen, die ein ,gemeinsames Leben“ (koinos bios) hinter Klostermauern führten, den „Koinobiten“. Das macht deutlich, dass die Leitfrage dieses Beitrags nicht eine Nischenproblematik war, sondern alle Lebensbereiche des spätantiken Christentums betraf.

Um 300 n. Chr. traten in Ägypten Menschen auf, die man bald als monachoi (,Vereinzelte“) bezeichnete. Diese „Mönche“ gingen dorthin, wo zuvor kein Mensch gewesen war oder jedenfalls nicht lange verweilen mochte: in die Wüste, das Reich der Dämonen, wo der Teufel vierzig Tage lang (erfolglos) Jesus Christus versucht hatte (Mt 4,1-11). Diesen Sieg galt es nachzuahmen, und dazu musste man alles hinter sich lassen, was die Konzentration auf Gott und damit die Widerstandsfähigkeit gegen die Dämonen stören konnte: die Pflege der äußeren Erscheinung, Sexualität und Familie, das Streben nach Karriere und sozialem Aufstieg - und auch den Erwerb von Bildung. Der berühmteste dieser Wüstenväter, Antonius (†356), wird von seinem Biographen Athanasius (†373) als gänzlich ungebildet dargestellt: Antonius verweigert schon als Kind den Schulbesuch, um nicht vom Kontakt mit der Welt beeinträchtigt zu werden, und dass er später Disputationen mit Sophisten, die ihn auf seinem Berg aufsuchen, souverän gewinnt, liegt daran, dass er von Jugend an ,unverbildet" (aplastos, ,ungeformt") ist und sich als ,Theodidakt", erweist, der direkt von Gott belehrt wurde, wie schon Paulus (1 Thess 4,9) den Christen angekündigt 
hatte (Athanasius, Vita Antonii 1,2; 66,2). Er erschien als Nachfolger der Apostel Petrus und Johannes, die für ,ungebildet und unkultiviert“ (agrammatoi kai idiotai) gehalten wurden, aber mit vollmächtiger und überzeugender Rede überraschten (Apg 4,13).

Das Bild des Antonius, der des Lesens und Schreibens nicht mächtig gewesen sei, aber die gesamte Bibel auswendig habe hersagen können (Athanasius, Vita Antonii 3,7) - Augustin war davon offensichtlich beeindruckt (De doctrina christiana prol. 4,8) -, ist in der Forschung lange Zeit für bare Münze genommen worden (vgl. Dörries 1966). Doch hat sich dies gewandelt: Unter Antonius' Namen sind Briefe überliefert, in denen er einer monastischen Gemeinschaft anthropologische Reflexionen auf dem Niveau der zeitgenössischen alexandrinischen Theologie darlegt (Rubenson 1995). Er war vielleicht kein Fachphilosoph, aber ein philosophischer Kopf! Die Vita Antonii berichtet bei näherem Hinsehen nicht von einem Mangel an Lese- und Schreibkompetenzen, sondern hebt ganz bewusst die fehlende Vertrautheit mit antiker, d.h. mit „falscher“ Religion kontaminierter Literatur hervor. Die Enthaltsamkeit betrifft also nicht jede, sondern nur die in die Irre führende Bildung. Und so wurde Antonius, der Philosoph sui generis, zum Exponenten einer monastischen Bildungsbewegung (vgl. Gemeinhardt 2013, S. $110 \mathrm{ff}$.).

Das frühe Mönchtum bildete schon bald Institutionen aus. Pachomius († 346) begründete in Oberägypten das Klosterwesen, das rasch auf Palästina, Syrien und Kleinasien und auf den Westen des Imperium Romanum ausstrahlte. In einem Kloster (monasterion) wurde die oben skizzierte Lebensweise des monachos in eine Form gebracht, die nicht nur für Hard-core-Eremiten zu bewältigen war, sondern für alle, die im Bewusstsein ihrer menschlichen Schwachheit ein geistliches Leben führen wollten. In diesem Leben spielte die Bibel eine zentrale Rolle, nicht nur durch Psalmen und Lesungen im Gottesdienst, sondern auch durch individuelle Lektüre - und dafür mussten Mönche lesen können; wenn sie diese Kompetenz beim Klostereintritt noch nicht mitbrachten, erhielten sie die nötige Unterweisung im internen Unterricht (Regula Pachomii 139; 140). Durch die Pflicht zum Umgang mit der Heiligen Schrift wurden Klöster zu Bildungsinstitutionen (wenn auch mit moderaten Ansprüchen). Im Abendland wurde die im 6. Jahrhundert verfasste Regula Benedicti zum Leitbild. Für Benedikt von Nursia $(\dagger 547)$ war es eine eigentlich nicht hinnehmbare Erfahrung, dass ein Mönch nicht willens oder fähig war, lesen zu lernen - eher zähneknirschend wurde konzediert, so einer möge sich dann eben mit Handarbeit beschäftigen (Regula Benedicti 48,15.23). Bemerkenswert ist, dass bereits in der pachomianischen Gemeinschaft und ausdrücklich auch in gallischen Klosterregeln Männer und Frauen solche Bildung empfangen sollten: „Sie sollen alle lesen lernen“, verfügte Bischof Caesarius von Arles († 542) für das von seiner Schwester geleitete Kloster (Regula virginum 18,7). Eine andere gallische Regel präzisiert, damit seien die Schriften der Bibel, nicht aber die „heidnischen Fabeln“ gemeint, die den Sinn der Hörenden nicht auferbauen, also nicht geistlich bilden würden (Regula Tarnantensis 9). Man musste Vorsicht walten lassen, denn so weit war die „Welt“ mit ihren literarischen Versuchungen dann doch nicht weg!

Die Verhältnisbestimmung von Bildung und Religion war im Mönchtum keineswegs unkompliziert, denn wenn man auch die Inhalte zurückwies, die in den Unterrichtsmaterialien der scholae publicae Verwendung fanden, so war es doch 
kaum möglich und auch gar nicht sinnvoll, auf das didaktische Handwerkszeug zu verzichten (vgl. Larsen und Rubenson 2018). Wenn in der Wüste religiöse Bildung vermittelt wurde, vollzog sich das in ähnlicher Form wie bei Augustin: als dialogisches Lernen, inspiriert und gefördert von Christus als dem wahren Lehrer, der menschliches Lehren nicht erübrigt, aber relativiert. Das zeigen die Apophthegmata Patrum, die „Sprüche der Väter“ (vgl. einführend Schulz und Ziemer 2010). Unter diesem Titel entstanden seit dem 5. Jahrhundert mehrere Sammlungen von Aussprüchen der großen Gestalten des frühen Eremitentums. Ein typisches Apophthegma hat eine knappe szenische Rahmung, in der einer der Väter (gelegentlich auch eine Wüstenmutter) um Belehrung gebeten wird; diese wird dann in Form eines prägnanten „Ausspruchs“, des „Apophthegma“, gegeben. Repräsentativ ist z.B. die folgende, Antonius zugeschriebene Aussage:

Einer fragte den Abbas Antonius: Was muss ich beachten, damit ich Gott gefalle? Und der Greis antwortete und sprach: Was ich dir auftrage, beachte: Wohin du auch gehst, habe immer Gott vor Augen! Und was du auch tust: Habe dafür ein Zeugnis aus der Schrift! Und an welchem Ort du auch sitzt, entferne dich nicht rasch wieder! Wenn du diese drei Dinge beachtest, wirst du gerettet (Apophthegmata Patrum Antonius 3). ${ }^{15}$

Diese Lehre - hier fokussiert auf die Orientierung auf Gott, auf die Bibel als Quelle entsprechender Verhaltensweisen und auf stabilitas loci anstelle des Suchens nach immer neuen Zielen - sollte nichts Geringerem als dem ewigen Heil der Seele dienen:

Derselbe sagte: Niemand kann unversucht in das Himmelreich eingehen. Nimm die Versuchungen weg, und niemand wird gerettet (Apophthegmata Patrum Antonius 5).

Die Autorisierung solcher Lehren erfolgte nicht durch ein kirchliches Amt, sondern durch geistliche Erfahrung: Ein „Greis“ (geron), einer, dem man Weisheit zuschrieb, war Antonius nicht wegen seines Lebensalters; die monastische Tradition kannte sogar manchen „Kindgreis“ (paidariogeron, puer senex), der schon in jungen Jahren so viel Erfahrung gesammelt hatte, dass er andere belehren konnte. ${ }^{16}$ Religion in der Wüste lehren konnte daher nur jemand, der damit Erfahrung gesammelt hatte, der also nicht nur Wissen erworben, sondern sich in Anfechtungen durch äußere und innere Versuchungen bewährt hatte. Es liegt auf der Hand, dass eine solche erfahrungsbasierte Autorisierung in Konkurrenz zum institutionalisierten Katechumenat geraten konnte (vgl. Gemeinhardt 2021a, S. 184-193).

Die Form der Belehrung, wie sie die Apophthegmata Patrum darstellen, hatte formal und auch sachlich mit dem Vorbild Jesu zu tun, besaß aber in den rhetorischen Schulübungen (Progymnasmata) eine wichtige Parallele: die „Chrie“, eine knappe Sentenz, die dazu diente, moralische Maximen zu memorieren (vgl. McVey 1998). Der schulische - nicht genuin christliche oder biblische - Bildungshintergrund war in der Wüste präsent, gelegentlich schien er sogar explizit durch:

15 Übersetzungen nach Schweitzer (2012).

16 Z. B. der palästinische Mönchsvater Sabas (6. Jh.; Kyrill von Skythopolis, Vita Sabae 11). 
Abbas Esaias sagte zu denen, die gut beginnen und den heiligen Vätern unterstellt sind: Die erste Färbung verliert sich nicht, wie beim Purpur. Und wie die jungen Zweige sich leicht drehen und biegen lassen, so auch die Anfänger in der Unterweisung (Apophthegmata Patrum Esaias 2).

Trotz der in den Apophthegmata Patrum vielfach formulierten Ablehnung klassischer Bildung erfolgte hier ein kaum verhüllter Rückgriff auf diese: Mit dem Bild, dass die erste Unterweisung unauslöschlich im Gedächtnis hafte wie die erste Färbung in der Purpurwolle, hatte auch der asketische Autor Hieronymus († 419) die Bedeutung (und Gefahr!) frühkindlicher Bildung herausgestellt - und dabei auf ein Bild des römischen Dichters Lukrez ( $\dagger$ um 55 v. Chr.) zurückgegriffen. ${ }^{17}$

Der spätantike christliche Diskurs über Bildung entkam also selbst in der Wüste nicht dem Paradox, dass religiöse Bildung nicht ohne säkulare Bildung zu haben war - und es scheint, als hätten die Wüstenväter dies überwiegend entspannt gesehen. Auch in der Wüste wurde Religionsdidaktik getrieben, und während von der Generation Augustins und Hieronymus' die Entwicklung einer alternativen christlichen Bildung oft gefordert, aber nie realisiert wurde, fand sie in Eremitenzellen und hinter Klostermauern ihren Platz: Das Mönchtum erscheint von hier aus sogar als Avantgarde spätantiker christlicher Bildung!

\section{Fazit und Ausblick}

Ist Religion lehrbar? Ziehen wir ein kurzes (und angesichts der Weite des Gegenstandes nur vorläufiges) Fazit: Religion ist - in der Sicht ihrer spätantiken christlichen Protagonisten - lehrbar unter Beteiligten, d.h. unter Empfangenden und Gebenden. Gelehrt wird sie angemessener Weise im Dialog, und dieser Dialog relativiert menschliche Autorität. Zwar gibt es Lehrer und Schüler, also asymmetrische Situationen der Bildungsvermittlung, aber diese sind umfangen von christlicher Gemeinschaft, die wiederum der antiken amicitia frappierend ähneln kann. In dieser Hinsicht gibt es übrigens (ohne dies hier näher ausführen zu können) auch Parallelen zum frühen Judentum: Die Rabbinen wollten keine Autodidakten, sondern Gemeinschaft von Lehrenden und Lernenden, und mahnten: „Schaff dir einen Lehrer und erwirb dir einen Kollegen!“ (Mischna Avot 1,6; vgl. Langer 2012). Lerngemeinschaften finden wir z. B. auch in der spätantiken platonischen Philosophie und im Islam; auch spielt hier die Liebe als Movens des Bildungsprozesses und als Basis einer „Ethik des Lehrens“ eine zentrale Rolle (vgl. Günther 2015, S. 124).

Um beim Christentum zu bleiben: Deutlich ist, dass religiöse Bildung - im Sinne des oben zitierten jesuanischen Taufbefehls - einen lebensgeschichtlichen Haftpunkt hat, nämlich die unmittelbare Vor- und Nachbereitung der Initiation, sich aber davor und danach auch über längere Phasen erstreckt, ja das ganze Leben umgreift

17 Hieronymus (Epistula 107,4,6; Adversus Rufinum 1,30); vgl. Lukrez (De rerum natura 6,1074-1077): An beiden Stellen ergänzt Hieronymus ein anderes Bild des Poeten Horaz (Epistula 1,2,69f.), wonach ein Gefäß den Geschmack und Geruch seines ersten Inhalts behalte; auch verwendet bei Hieronymus (Epistula 10,3,3). 
(oder umgreifen kann). Und ebenso sollten die diskutierten Quellen gezeigt haben, dass über Unterschiede und sogar Grenzziehungen zwischen religiöser und nichtreligiöser (,heidnischer“) Bildung engagiert diskutiert wurde, dass aber auch für religiöse Zwecke pädagogische und didaktische Kompetenzen eingesetzt wurden, die sich nicht einer religiösen Zielbestimmung verdankten. Das kann auch nicht überraschen: Religion war ein Teil des Lebens, sie war verwoben mit anderen sozialen und kulturellen Praktiken, und daher konnte sich christliche Bildungskritik und -rezeption nur innerhalb des Rahmens bewegen, den die griechisch-römische Welt bot. Sich ostentativ von deren Idealen zu distanzieren war Teil des Spiels und doch auch mehr als nur ein Spiel; denn unter den vielen Göttern den einen und dreieinigen Gott als wahren Retter anzuerkennen war für christliche Identität grundlegend. Aber fest zu glauben hieß keineswegs, borniert zu sein!

Entscheidend dürfte Folgendes sein: Dialogisches Lernen ist in christlicher Sicht dadurch charakterisiert, dass der erste und eigentliche Lehrer Gott bzw. Christus ist. Das setzt jeder Operationalisierungslogik Grenzen: Menschen können religiöse Bildung vermitteln und erwerben, doch wird das menschliche Tun durch den Bezug auf den göttlichen Lehrer heilsam relativiert. Und umgekehrt: Der Mensch als „bildsames Wesen“ (Benner 2014) ist für religiöse Erfahrung offen und zu deren reflexiver Verarbeitung befähigt. Im Dialog der Lehrenden und Lernenden - sei er hierarchisch oder auf Augenhöhe angelegt - spielt immer noch eine dritte Instanz eine Rolle, die menschliches Bildungshandeln zugleich motiviert und relativiert. Diese Triangulation, die Bildung als zwischenmenschliches Geschehen von ihrem Grund unterscheidet, beides aber miteinander verbindet, stand auch Pate für den deutschen Begriff „Bildung“ in der Mystik und für seine Wirkungen in der Theologie und Pädagogik der Aufklärung. Religion ist also etwas anderes als Bildung, dieser aber nicht fremd - und umgekehrt.

Gibt all das auch heute noch zu denken? Ich hoffe doch. Die Religionspädagogik des 20. Jahrhunderts hat die Lehr- und Lernbarkeit von Religion reflektiert und (mit Bernd Schröder) ein ,religionspädagogisches Paradox“ identifiziert: Insofern Glaube ein Geschenk Gottes ist, bleibt er unverfügbar und kann nicht durch Bildungsprozesse ,produziert“" werden; insofern Glaube aber auf Verstehen zielt, ist er auf Bildung angewiesen (Schröder 2012, S. 212 f.). Dabei wurde im religionspädagogischen Diskurs über ,die Lehrbarkeit“ von Religion gestritten, „die Lernbarkeit christlicher Religion indes nicht in Abrede“ gestellt: „Ob Gott oder ein menschlicher Pädagoge als Lehrer gedacht wird - das menschliche Individuum lernt" (Schröder 2012, S. 204). Dass es hingegen von anderen über den Glauben belehrt werden könne, wurde immer wieder problematisiert. Die Sensibilität dafür, dass gelehrt werden muss, was im Kern nicht gelehrt werden kann, lässt sich bis zum frühen Christentum zurückverfolgen - und auch das Gespür dafür, dass Religion, mit Schleiermacher $(1799=1969$, S. 36), „Sinn und Geschmack fürs Unendliche“ und insofern von anderen möglichen Gegenständen pädagogischen Handelns kategorial verschieden ist.

Darum ist der schulische Religionsunterricht auf den ersten Blick kein Fach wie jedes andere, denn er setzt eine Beteiligtenperspektive voraus, insofern er - im deutschen Sprachraum - ausdrücklich in konfessioneller Perspektive erteilt wird. Darum geht es hier aber nicht weniger als in anderen Fächern um Bildung, und dies in 
großer Offenheit. Denn das, was im Religionsunterricht vermittelt wird, ist auch für nicht direkt Beteiligte ein Angebot zur Deutung von Selbst und Welt: Glaube und Handeln aus religiöser Motivation sollen ja gerade in ihrer Logik transparent und damit rational nachvollziehbar gemacht werden. Damit ist nicht die Forderung nach einer Konversion verbunden, wohl aber die Einladung, sich auf die Logik religiöser Bildung einzulassen. Dies bedingt zugleich die Bereitschaft der Lehrenden, sich auf eine offene Rezeptionssituation einzustellen, ja sogar auf den Streit der Deutungsangebote. Zugespitzt gesagt: Wenn das christliche Bildungsbemühen sich - nach Jesu Anweisung an seine Jünger - an alle Menschen richtet und religiöse Bildung stets an die Eigenaktivität des Subjekts gebunden ist, wird unter geschichtlichen Bedingungen eine Vielfalt von Rezeptionsweisen entstehen. In anderen Worten: Religiöse Bildung, die sich selbst ernst nimmt, muss pluralismusfähig sein.

Diese Pluralitätsfähigkeit hat sich christliche Theologie erarbeiten müssen; sie war in der hoch kompetitiven Situation der Spätantike keineswegs selbstverständlich - und ist doch ohne sie nicht zu denken. Das heißt nicht, in Abrede zu stellen, dass religiöse Bildung historisch mit Strategien der Inklusion und Exklusion operiert hat; doch soll behauptet werden, dass dies nicht zwangsläufig der Fall sein muss. Religion und Bildung gehörten im Christentum von Anfang an zusammen. Und auch wenn dieses Miteinander oft spannungsreich war, meine ich doch, dass darin einer der Faktoren zu sehen ist, weshalb das Christentum seine eigene Rationalität in wechselnden Kontexten neu bestimmen, behaupten und transformieren konnte. Das galt sogar für seit dem Mittelalter entstehenden Universitäten, an denen auch heute noch nicht nur, aber nicht zuletzt die Theologie kritisch und selbstkritisch das Bewusstsein für die Unverfügbarkeit von Bildungsprozessen offenhalten muss. Über Fakultäten und Disziplinen hinweg über Religion als Gegenstand und Grund von Bildung ins Gespräch zu kommen mag je und je sogar dazu beitragen, dass Lehrende und Lernende dabei zu gemeinschaftlicher hilaritas gelangen.

Danksagung Herrn Vinzent Wiedemann danke ich für kritische Lektüre und hilfreiche Nachfragen.

Förderung Der vorliegende Beitrag basiert auf Forschungen im Rahmen des DFG-geförderten SFB 1136 „Bildung und Religion in Kulturen des Mittelmeerraums und seiner Umwelt von der Antike bis zum Mittelalter und zum Klassischen Islam“.

Funding Open Access funding enabled and organized by Projekt DEAL.

Open Access Dieser Artikel wird unter der Creative Commons Namensnennung 4.0 International Lizenz veröffentlicht, welche die Nutzung, Vervielfältigung, Bearbeitung, Verbreitung und Wiedergabe in jeglichem Medium und Format erlaubt, sofern Sie den/die ursprünglichen Autor(en) und die Quelle ordnungsgemäß nennen, einen Link zur Creative Commons Lizenz beifügen und angeben, ob Änderungen vorgenommen wurden.

Die in diesem Artikel enthaltenen Bilder und sonstiges Drittmaterial unterliegen ebenfalls der genannten Creative Commons Lizenz, sofern sich aus der Abbildungslegende nichts anderes ergibt. Sofern das betreffende Material nicht unter der genannten Creative Commons Lizenz steht und die betreffende Handlung nicht nach gesetzlichen Vorschriften erlaubt ist, ist für die oben aufgeführten Weiterverwendungen des Materials die Einwilligung des jeweiligen Rechteinhabers einzuholen.

Weitere Details zur Lizenz entnehmen Sie bitte der Lizenzinformation auf http://creativecommons.org/ licenses/by/4.0/deed.de.

Interessenkonflikt P. Gemeinhardt gibt an, dass kein Interessenkonflikt besteht. 


\section{Literatur}

\section{Verwendete Literatur}

Becker, M. (2019). Zwischen Gelehrsamkeit und Angleichung an Gott. Bildung in der spätantiken Philosophie. In P. Gemeinhardt (Hrsg.), Was ist Bildung in der Vormoderne? (S. 205-228). Tübingen: Mohr Siebeck.

Benner, D. (2014). Bildung und Religion. Nur einem bildsamen Wesen kann ein Gott sich offenbaren. Paderborn: Schöningh.

Döpp, S., \& Geerlings, W. (Hrsg.). (2002). Lexikon der antiken christlichen Literatur (3. Aufl.). Freiburg: Herder.

Dörries, H. (1966). Die Vita Antonii als Geschichtsquelle. In H. Dörries (Hrsg.), Gesammelte Studien zur Kirchengeschichte des 4. Jahrhunderts (Wort und Stunde, Bd. I, S. 145-224). Göttingen: Vandenhoeck \& Ruprecht.

Dressler, B. (2006). Unterscheidungen. Religion und Bildung. Leipzig: Evangelische Verlagsanstalt.

Dujarier, M. (1979). A history of the catechumenate. The first six centuries. New York: Sadlier.

Gavrilyuk, P. L. (2007). Histoire du catéchuménat dans l'église ancienne. Paris: Éditions du Cerf.

Gemeinhardt, P. (2007). Das lateinische Christentum und die antike pagane Bildung. Tübingen: Mohr Siebeck.

Gemeinhardt, P. (2008). Dürfen Christen Lehrer sein? Anspruch und Wirklichkeit im christlichen Bildungsdiskurs der Spätantike. Jahrbuch für Antike und Christentum, 51, 25-43.

Gemeinhardt, P. (2013). Antonius: Der erste Mönch. Leben - Lehre-Legende. München: C. H. Beck.

Gemeinhardt, P. (Hrsg.). (2019). Was ist Bildung in der Vormoderne? Tübingen: Mohr Siebeck.

Gemeinhardt, P. (2020a). Ist das Christentum eine Bildungsreligion? Beobachtungen zu Bildungsprozessen und -zielen in der frühchristlichen Apologetik und Katechetik. In C. Müller \& G. Förster (Hrsg.), Augustinus als Pädagoge und als Sprachtheoretiker (S. 17-51). Würzburg: Echter.

Gemeinhardt, P. (2020b). Teaching the faith in early Christianity. Divine and human agency. Vigiliae Christianae, 74, 129-164.

Gemeinhardt, P. (2021a). Tradition, Kompetenz und Charisma: Streiflichter auf das Spannungsfeld von Autorität und Bildung in spätantiken Religionskulturen. In P. Gemeinhardt \& T. S. Scheer (Hrsg.), Autorität im Spannungsfeld von Bildung und Religion (S. 161-201). Tübingen: Mohr Siebeck.

Gemeinhardt, P. (2021b). Bildung - Theologie - Bildungsreligion: Christentumsgeschichtliche Perspektiven. In B. Schröder (Hrsg.), Bildung (S. 65-103). Tübingen: Mohr Siebeck.

Günther, S. (2015). Auf der Suche nach dem Elixier der Glückseligkeit. Konzeptionen rationaler und spiritueller Bildung im Klassischen Islam. In P. Gemeinhardt \& T. Georges (Hrsg.), Theologie und Bildung im Mittelalter (S. 111-128). Münster: Aschendorff.

Harmless, W. (2014). Augustine and the catechumenate (2. Aufl.). Collegeville: Liturgical Press.

Kinzig, W. (2019). Formation des Glaubens. Didaktische und liturgische Aspekte der Rezeption altkirchlicher Symbole in der lateinischen Kirche der Spätantike und des Frühmittelalters. In U. Heil (Hrsg.), Das Christentum im frühen Europa. Diskurse - Tendenzen - Entscheidungen (S. 389-432). Berlin: de Gruyter.

Langer, G. (2012). Menschen-Bildung. Rabbinisches zu Lernen und Lehren jenseits von PISA. Wien: Böhlau.

Larsen, L.I., \& Rubenson, S. (Hrsg.). (2018). Monastic education in late antiquity: the transformation of classical Paideia. Cambridge: Cambridge University Press.

Markschies, C. (2007). Kaiserzeitliche christliche Theologie und ihre Institutionen. Prolegomena zu einer Geschichte der antiken christlichen Theologie. Tübingen: Mohr Siebeck.

McVey, K. (1998). Chreia in the desert: rhetoric and the bible in the Apophthegmata Patrum. In A. J. Malherbe, F. W. Norris \& J. W. Thompson (Hrsg.), The early church in its context. Essays in honour of Everett Ferguson (S. 245-255). Leiden: Brill.

Metzger, M., Brakmann, H., \& Drews, W. (2004). Katechumenat. In Reallexikon für Antike und Christentum (Bd. 20, S. 497-574).

Patzold, S. (2015). Correctio an der Basis: Landpfarrer und ihr Wissen im 9. Jahrhundert. In J. Becker, T. Licht \& S. Weinfurter (Hrsg.), Karolingische Klöster. Wissenstransfer und kulturelle Innovation (S. 227-254). Berlin: de Gruyter.

Paul, E. (1993). Antike und Mittelalter (Geschichte der christlichen Erziehung, Bd. I). Freiburg: Herder.

Reil, E. (1989). Aurelius Augustinus. De catechizandis rudibus. Ein religionsdidaktisches Konzept (Bd. 33). St. Ottilien: EOS. 
Rubenson, S. (1995). The letters of St. Antony. Monasticism and the making of a saint. Minneapolis: Fortress Press.

Sautermeister, J., \& Zwick, E. (Hrsg.). (2019). Religion und Bildung: Antipoden oder Weggefährten? Diskurse aus historischer, systematischer und praktischer Sicht. Paderborn: Schöningh.

Schleiermacher, F. D. E. (1969). Über die Religion. Reden an die Gebildeten unter ihren Verächtern (1799). Stuttgart: Reclam.

Schröder, B. (2012). Religionspädagogik. Tübingen: Mohr Siebeck.

Schulz, G., \& Ziemer, J. (2010). Mit Wüstenvätern und Wüstenmüttern im Gespräch. Zugänge zur Welt des frühen Mönchtums in Ägypten. Göttingen: Vandenhoeck \& Ruprecht.

Studer, B. (1996). Die Kirche als „Schule des Herrn“ bei Augustinus von Hippo. In G. Schöllgen (Hrsg.), Stimuli. Exegese und ihre Hermeneutik in Antike und Christentum. FS E. Dassmann (S. 486-498). Münster: Aschendorff.

Vössing, K. (1997). Schule und Bildung im Nordafrika der römischen Kaiserzeit. Brüssel: Latomus.

Vössing, K. (2003). Die Geschichte der römischen Schule - ein Abriß vor dem Hintergrund der neueren Forschung. Gymnasium, 110, 455-497.

\section{Quellenverzeichnis}

Barthold, C. (Übers.) (2011). Origenes: Contra Celsum. Gegen Celsus (Bd. 1, Hrsg. von M. Fiedrowicz). Freiburg: Herder.

Bernhart, J. (Übers.) (1987). Augustinus: Confessiones - Bekenntnisse. Frankfurt a. M.: Insel.

Geerlings, W. (Übers.) (1991). Traditio Apostolica. In W. Geerlings \& G. Schöllgen (Hrsg.), Didache und Traditio Apostolica (S. 212-313). Freiburg: Herder.

Haeuser, P. (Übers.) (1922). Cyrillus von Jerusalem, Katechesen. Kempten: Kösel \& Pustet.

Pollmann, K. (Übers.) (2002). Augustinus: Die christliche Bildung (De doctrina Christiana). Stuttgart: Reclam.

Schweitzer, E. (Übers.) (2012). Das Alphabetikon - Die alphabetisch-anonyme Reihe (Apophthegmata Patrum, Bd. I). Beuron: Beuroner Kunstverlag.

Steinmann, W., \& Wermelinger, O. (Übers.) (1985). Aurelius Augustinus: Vom ersten katechetischen Unterricht. München: Kösel.

Ulrich, J. (Übers.) (2021). Justin, Apologiae. Apologien. Freiburg: Herder. 